\title{
CYCLIC RESPONSE OF A STEEL BEAM-TO-COLUMN CONNECTIONS - AN EXPERIMENTAL AND NUMERICAL STUDY
}

\author{
G.C. $\operatorname{Manos}^{1}$, A. Nalmpantidou ${ }^{2}$, V. Kourtides ${ }^{3}$, A. Anastasiadis ${ }^{3}$ \\ ${ }^{1}$ Professor and Director of the Lab. of Strength of Materials and Structures, Aristotle University \\ e-mail: gcmanos@civil.auth.gr \\ ${ }^{2}$ Postgraduate student, Lab. of Strength of Materials and Structures, Aristotle University \\ e-mail: a.nalbantidou@gmx.de \\ ${ }^{3}$ Dr. Civil Engineer, Research Ass., Lab. of Strength of Materials and Structures, Aristotle University \\ e-mail: anastasiadisa@hol.gr
}

Keywords: Steel beams, steel beam-to-column connection, cyclic response, plastic hinge, low-fatigue tests, strain rate effects, earthquake performance of steel connection

\begin{abstract}
The cyclic response of steel beam-to-column connections under a cyclic sinusoidal loading history was studied. The experimental investigation included two full-scale specimens having a cross section of IPE240 and a height of $1.0 \mathrm{~m}$. This part of the specimen was rigidly connected to a much stiffer steel beam thus representing a steel beam-to-column connection under investigation. These specimens were tested to failure being subjected to a cyclic sinusoidal point load of continuously increasing amplitude that produced a similarly varying bending moment at the steel beam-to-column connection. Instrumentation was provided to record both the applied moment as well as the deformation of the specimen in the region of the connection in terms of plastic hinge rotation. Each specimen experienced yielding and plastic rotation in this region that resulted initially in a plastic stable response till the final damage, which occurred either in the form of local instability of the flanges or fracture. The response of this beam-to-column connection was numerically simulated employing commercial software that tried to reproduce all the stages of the observed non-linear response. By comparing the numerical predictions with the measured response the success of the employed numerical simulation could be examined. The employed numerical simulation could yield realistic predictions of the limit state local buckling of the flanges of the used IPE 240 cross section as well as good agreement with the observed measured response, in terms of flexural capacity, energy dissipation and deterioration characteristics, provided that the material model in this numerical simulation was equipped with the "proper" parameters.
\end{abstract}




\section{INTRODUCTION}

Steel frame structures are commonly used in seismic areas because of their excellence on seismic resistance. This type of structures are usually composed of moment resistant frames whereby the performance of beam-to-column connections are of particular importance especially when these structures are subjected to strong earthquake ground motions $[1,2,5]$. Usually these connections undergo large moment reversals during the earthquake excitation, thus it is important to exhibit stable plastic flexural response in order to provide dissipative capability, without the development of undesired damage patterns, and in this way prevent any kind of local or global instability. However, two recent earthquakes, in Northridge, California 1994 and Kobe Japan 1995, revealed the vulnerability of this type of construction. Premature cracks were observed at welded connections of steel beam-to-column joints leading to brittle failure without prior warning (obvious yielding) at the area around the weld. Such a failure mode or the development of local buckling when beam-to-column connections undergo lowcycle fatigue seismic-type of loading is of particular research interest [3, 4, 9, 14].

The fatigue life of steel beam-to-column joints depends on the amplitude of the imposed loading cycles and the hysteretic behavior of the constituent material that is linked with the amplitude and rate of the developing plastic strains. In order to investigate the fatigue life of steel beam-to-column joints several fatigue testing investigations have been proposed leading to tests that can be categorized in two groups. Testing regimes in which the elastic strain amplitude is higher than the plastic strain amplitude and testing regimes in which the plastic strain dominates. The first type of tests is generally referred as high cycle fatigue (HCF); these tests typically involve more than $10^{6}$ cycles to cause failure. The second type of tests is named low cycle fatigue (LCF) test and the number of cycles to failure generally ranges from $10^{2}$ to $10^{4}$ cycles and this is usually employed for testing the seismic performance of steel beam-to-column joints $[6,8,11,12,15]$.

The current work studies the performance of steel beam-to-column joints first by examining steel beam-to-column welded joint specimens at the laboratory subjecting them to cyclic seismic-type low-cycle fatigue loading as is explained in section 2. Next, the performance of the same specimens is simulated numerically. Similar studies have been performed in the past by other researchers. Despite the fact that many similar experiments have been performed in the past $[6,8]$, the current experimental investigation was considered as necessary in order to provide at first hand all the information required for the numerical analyses. That is, the exact geometric and material characteristics of the specimens as well as specific information on the type of loading conditions and on the measured response. The numerical simulation is performed utilizing a commercial software package that is thought to be suitable to simulate the observed response and the non-linearities that developed and included large plastic strains and local instabilities.

\section{MATERIALS AND EXPERIMENTAL SETUP}

\subsection{Tested specimens}

Two full-scale specimens have been constructed with prototype steel cross sections in order to form a beam-to-column joint. Both specimens were of the same geometry and used as the beam part a IPE 240 steel section and as the column part a HEA 300 steel section. These two parts were welded together, as shown in figures 1 and 2, to form the beam-to-column connection to be tested. 
In order to confine the damaged region, where large strains were expected to develop thus making this part unusable, it was decided to weld the IPE 240 beam to a steel base plate having a thickness of $20 \mathrm{~mm}$. This steel plate in turn was bolted to the HEA 300 column (figure 1). Thus, after the end of the test sequence, the IPE 240 part with the steel base plate could be unbolted and removed from the HEA 300 column, which could be used again. Furthermore, in order to avoid the brittle type of failure at the welds joining the IPE 240 beam part with the base steel plate two $20 \mathrm{~mm}$ thick steel stiffeners of triangular shape (with a height of $160 \mathrm{~mm}$ and width of $170 \mathrm{~mm}$ ) were welded at either side of the IPE 240 section connecting its flanges with the base plate as shown in figures 1 and 2. In this way, the region of the IPE 240 beam to be plastified was removed from the welded region to the region above these stiffeners, thus providing a controlled beam-to-column connection to be investigated. The height of the IPE 240 beam part was $1200 \mathrm{~mm}$ allowing on one hand to construct the beam-to-column connection in the described way and on the other hand to connect its upper part with the forcing fixtures that were provided by a double acting hydraulic actuator. This actuator was controlled by a servo-hydraulic digital electronic controller providing the desired imposed cyclic horizontal displacement amplitude at a distance of $1000 \mathrm{~mm}$ from the base plate (see figure 2).
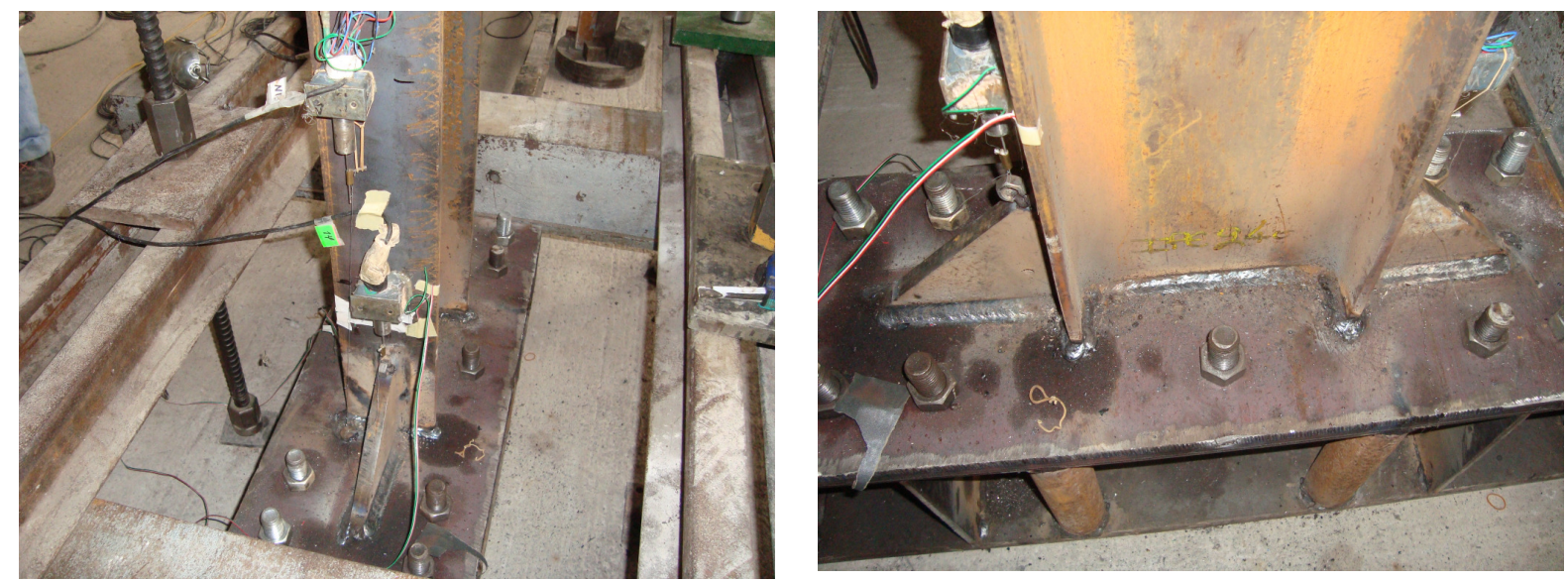

Figure 1. Details of the tested beam-to-column joint specimens

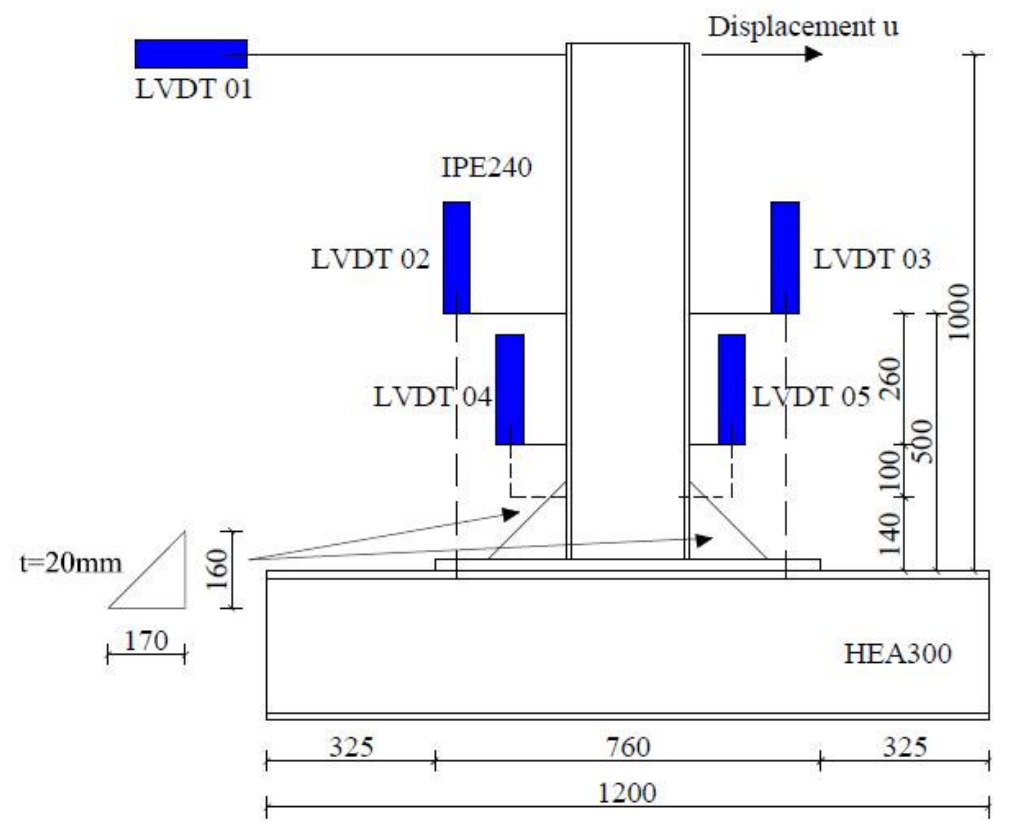

Figure 2. Experimental set-up with the geometry of the specimens, the instrumentation and loading 
Two IPE 240 beam-to-column connection specimens, namely Tbeam- 2 and Tbeam-4, were tested as shown in figures 2 and 3 . Both specimens were of the same overall geometry having webs with thickness equal to $6.5 \mathrm{~mm}$. The flange thickness varied from $9.16 \mathrm{~mm}$ to $9.36 \mathrm{~mm}$ and from $9.5 \mathrm{~mm}$ to $9.7 \mathrm{~mm}$ for specimens Tbeam-2 and Tbeam- 4 , respectively.

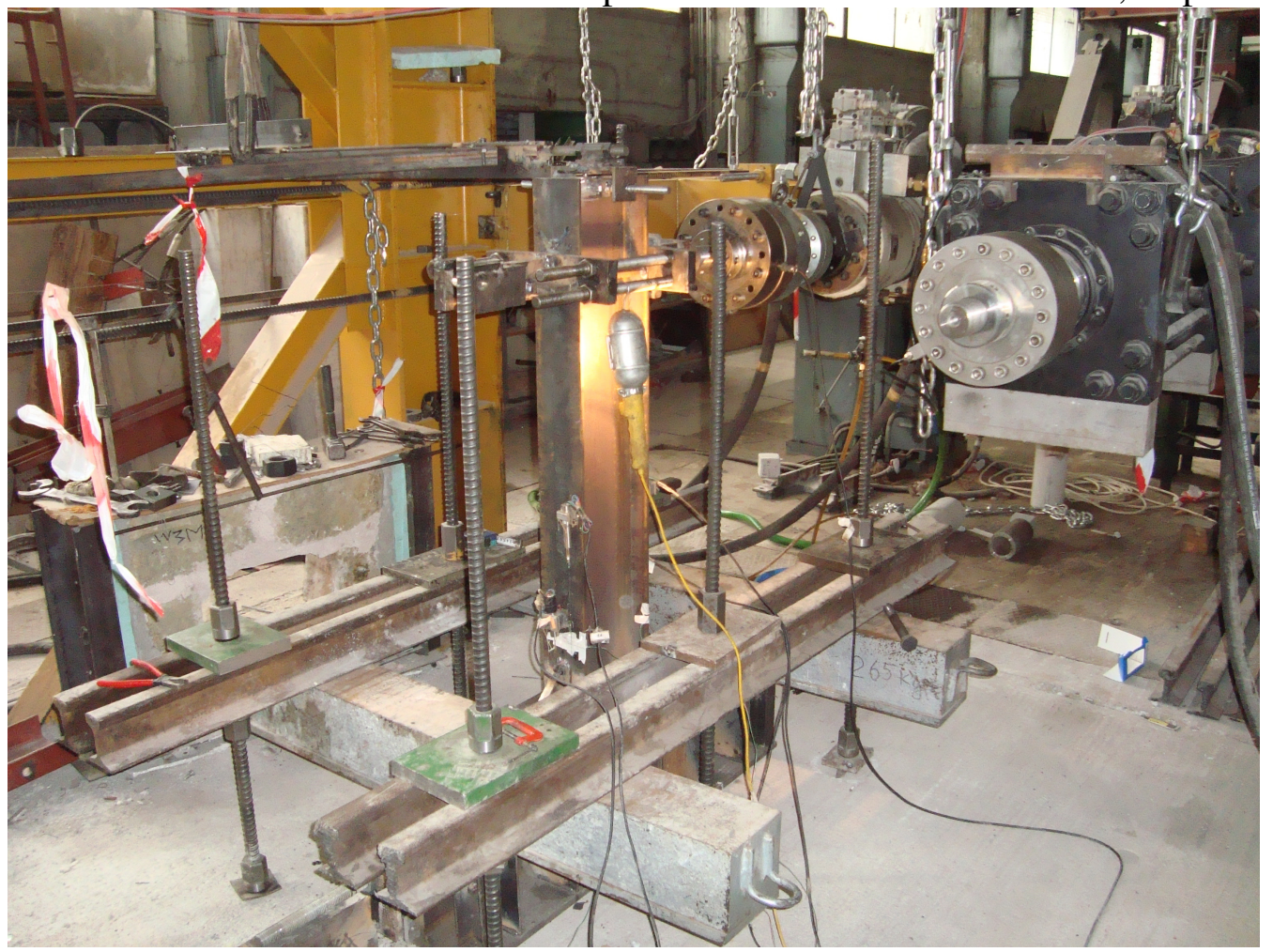

Figure 3: Experimental setup with the specimen being rigidly attached on the strong floor of the Laboratory of Strength of Materials and Structures of Aristotle University.

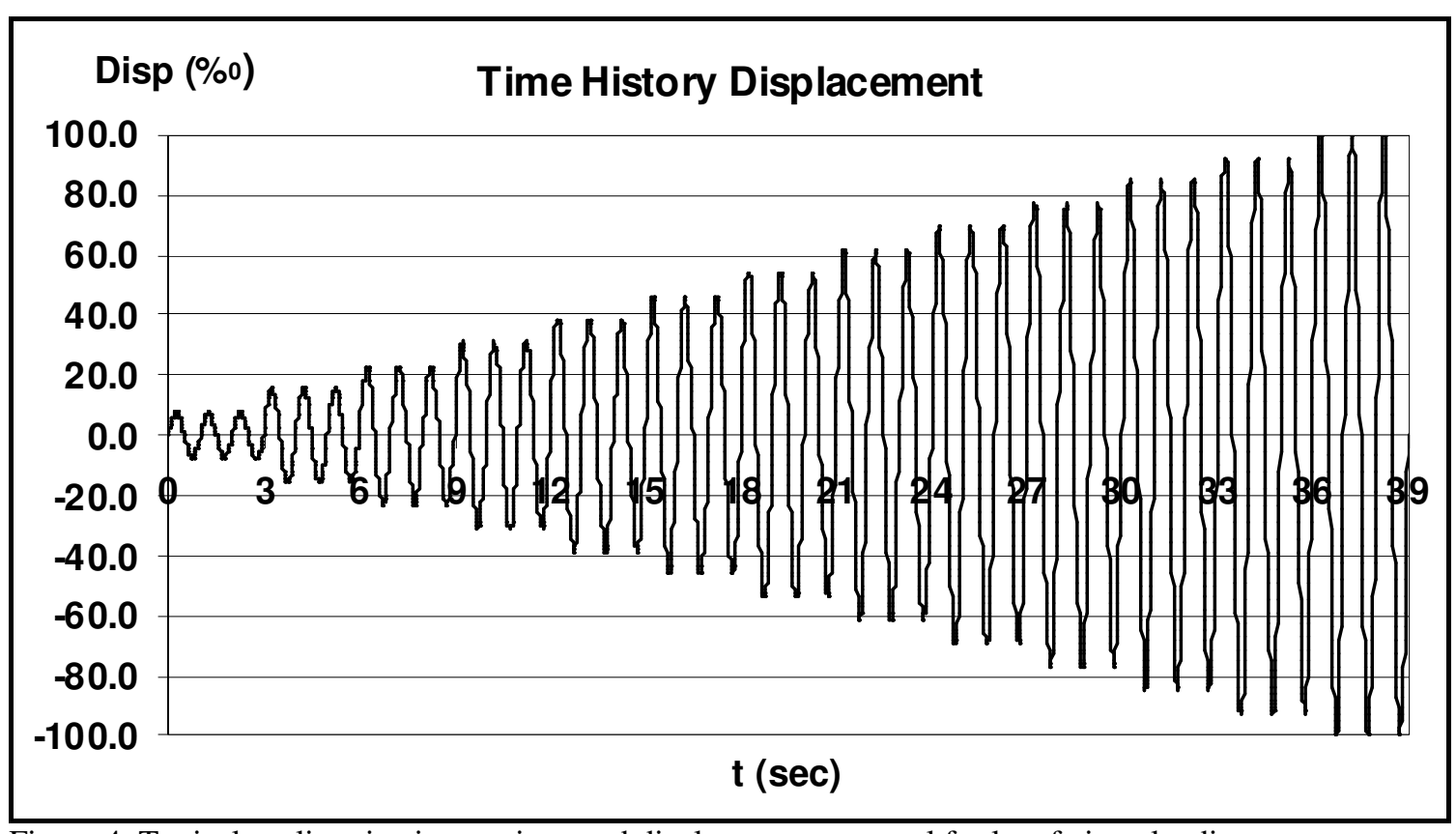

Figure 4. Typical cyclic seismic-type imposed displacement protocol for low fatigue loading 


\subsection{Loading arrangement}

The tested specimens were subjected to a cyclic seismic-type loading arrangement. An extensive discussion on the loading protocols was presented by Krawinkler [11]. This is a very important and difficult issue as on one hand it is believed that it influences the resulting performance and on the other hand it poses demands on the capabilities of the experimental facility $[6,7,8,11,12,15]$. As this work at its present stage aims to produce results that will be utilized for validating the numerical capabilities of existing software rather than checking the validity of code provisions the used loading protocol does not comply exactly with ones suggested by Krawinkler and other researchers [6, 7, 11, 15]. Instead, a series of sinusoidal horizontal cyclic displacements were used and were imposed on the top of the IPE 240 beam part, as shown in figures 2 and 3. Both specimens were rigidly attached on the strong floor of the Laboratory of Strength of Materials and Structures of Aristotle University. The hydraulic actuator was attached on the strong metal frame which together with the strong floor forms this experimental facility. The imposed horizontal displacement and the resulting horizontal load leads to the development of a bending moment at the beam-to-column connection which, if of sufficient amplitude, is expected to bring this part of the beam-to-column connection to a certain limit state condition, as will be explained in section 3. The imposed horizontal displacement in amplitude and frequency content is controlled by the electronic servo-controller. A relatively simple imposed displacement protocol is depicted in figure 4 . It consists of thirteen (13) groups of 3 full sinusoidal cycles. In each group the imposed maximum displacement amplitude remains constant and keeps gradually increasing from one group to the next in a prescribed manner, as a percentage of the maximum target displacement. The difficulty in imposing this protocol accurately lies on the fact that the target displacement is known at best through predictions that may differ considerably from the actual target displacement that, when imposed, will produced the desired phenomena. The protocol depicted in this figure was adopted in the current study in a general sense. That is, the amplitude of the imposed horizontal displacement kept increasing from one group to the next till the plastification of the tested specimens led to some type of failure, as will be described in section 3. One distinct difference in the followed loading protocol for the specimen Tbeam- 2 is the fact that the frequency content was relatively high $(0.1 \mathrm{~Hz}-1.0 \mathrm{~Hz})$ as to allow this test to be characterized as a dynamic test accompanied with medium level of strain rate. On the contrary, the loading protocol for Tbeam-4 specimen was relatively low $(0.003 \mathrm{~Hz}$ to $0.03 \mathrm{~Hz})$ that is characterized as cyclic test of relatively low level strain rate.

\subsection{Instrumentation}

Instrumentation was provided capable to record the dynamic response of the specimen at the top in terms of imposed horizontal displacement (LVDT 01, figure 2) and horizontal force. In addition, four displacement transducers (LVDT 02, LVDT 03, LVDT 04 and LVDT 05) were attached to the specimen in order to record the relative vertical displacement of two sets of horizontal cross sections of each specimen near the plastification zone (figures 1,2 and 3). The first set of horizontal cross sections was that at the base, where the IPE 240 part was welded at the base steel plate) and the one 500mm higher. Utilizing LVDT 02 and LVDT 03 the relative elongation or shortening between these two cross sections of the specimen was measured at the flanges of both sides of the IPE 240 section. The second set of horizontal cross sections was that at $160 \mathrm{~mm}$ from the base, where the triangular stiffeners ended, and the one 100mm higher. Utilizing LVDT 03 and LVDT 04 the relative elongation or shortening between these two cross sections of the specimen was measured at the flanges of both sides of 
the IPE 240 section. In this way, it became possible to deduce from the measured response the rotation between the first and the second set of these horizontal cross sections. The second set of horizontal cross sections is of particular importance as the highly plastified region (plastic hinge), which was expected to develop during the experimental sequence, lies between the horizontal cross-sections of the second set. Indeed, the used instrumentation was able to capture the plastification of this region from the initial stages of yielding till the final stages of local instability. In addition, a set of strain gauges was also provided in order to control in a different way the initiation and development of plastification in this zone.

Figures 5 and 6 depict the instrumentation of this zone near the beam-to-column joint. The used hydraulic actuator can also be seen in figure 5 whereas in figure 6 the vertical displacement transducer utilized to measure the relative elongation or shortening between two horizontal cross sections above the stiffener having a distance between them equal to $100 \mathrm{~mm}$ can also be seen (second set of horizontal cross sections).

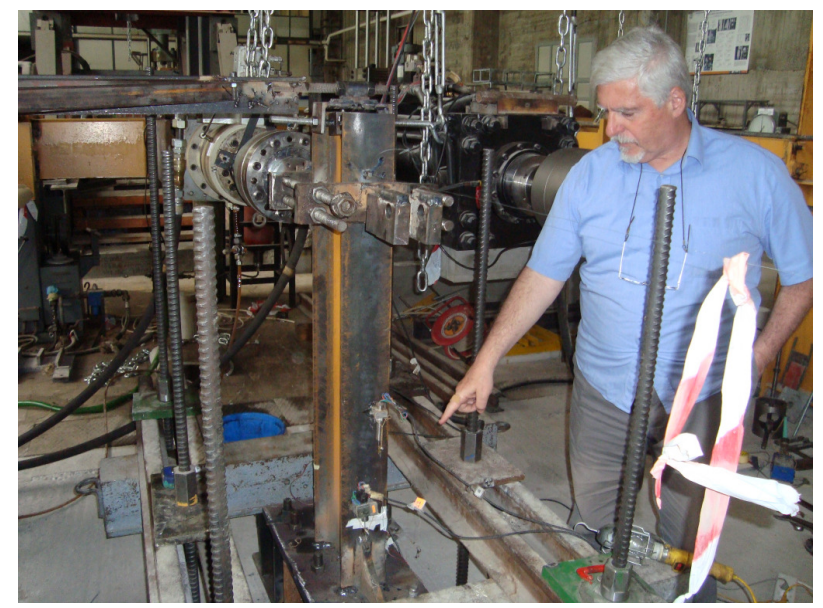

Figure 5. Instruments located near the beam-to-column joint.

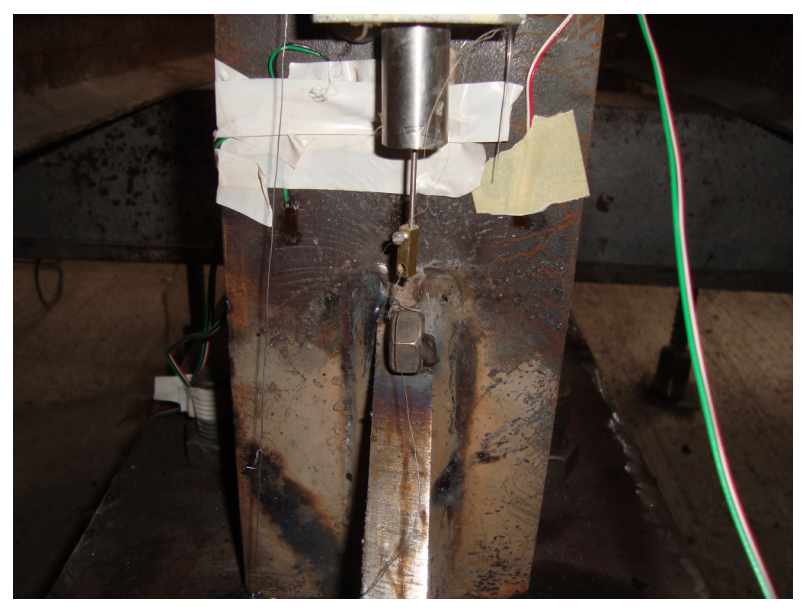

Figure 6. Measuring the relative elongation/shortening between two horizontal cross sections above the stiffener

\subsection{Steel tensile properties}

In order to input the proper information related to the steel characteristic in the finite element program monotonic tensile tests were performed with coupons taken from both specimens. These coupons were taken from the flanges of the specimens after the completion of the relevant test sequence. In order to measure the material properties of the virgin steel prior to any plastification and permanent straining these coupons were taken from the upper part of the IPE 240 beam sections near the section where the imposed displacement was applied The corresponding results are shown in figures 7 and 8 .

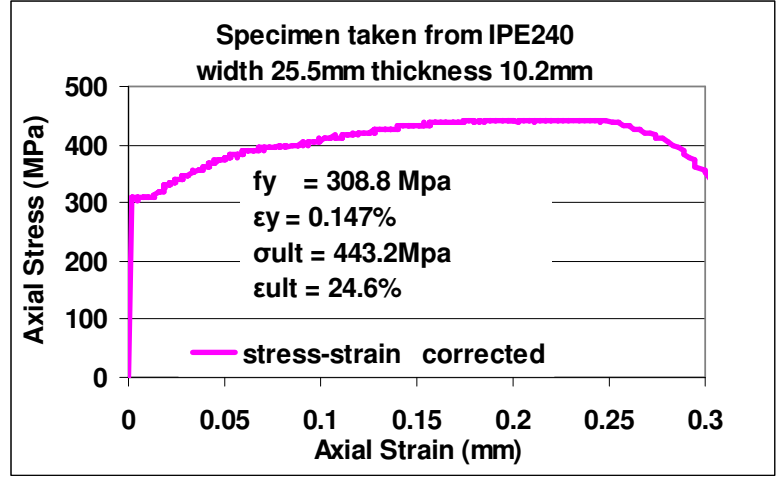

Figure 7. Tensile properties sample 1

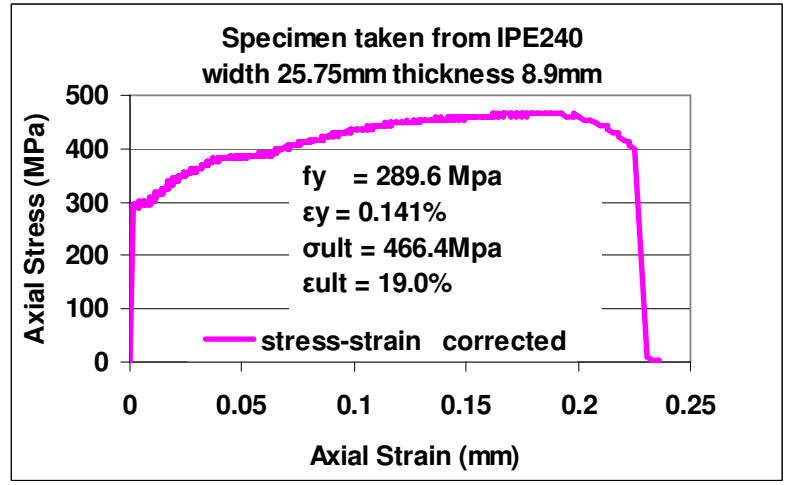

Figure 8. Tensile properties sample 2 


\section{OBSERVED PERFORMANCE AND CORRESPONDING MEASUREMENTS.}

\subsection{Observed over-all performance and actual damage}

Each specimen experienced plastic rotation above the stiffeners that was captured by the employed instrumentation. Despite the fixity of the attachment of the specimens on the strong floor part of the imposed horizontal displacement was consumed by the rotation of the specimen at its fixed base as well as of the elastic response along the height apart from the plastic rotation that developed at the desired location above the stiffeners. Fortunately, despite the limitations of the capabilities of the experimental facility it was possible to attain the plastic limit state for both studied specimens and produce the expected form of damage. This is depicted in figures 9 and 10 for specimen TBeam- 2 and figure 11 for specimen Tbeam-4.

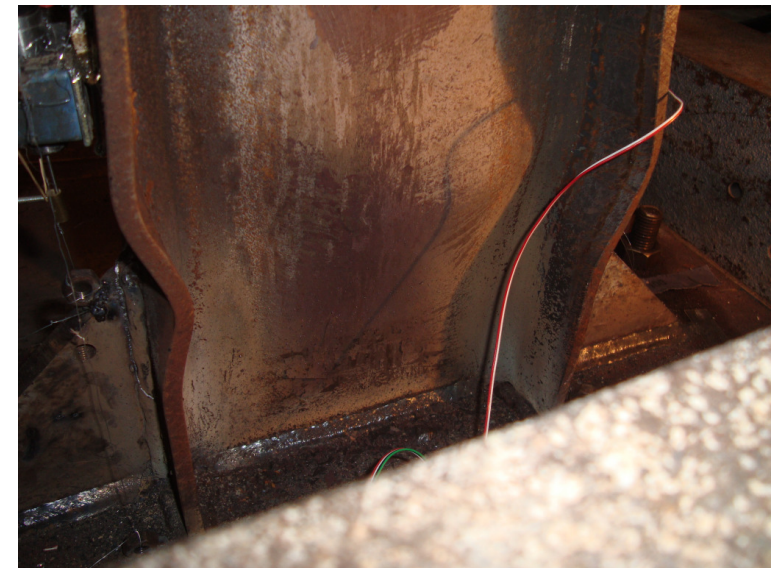

Figure 9. Plastification of specimen TBeam-2 at the end of Test-12

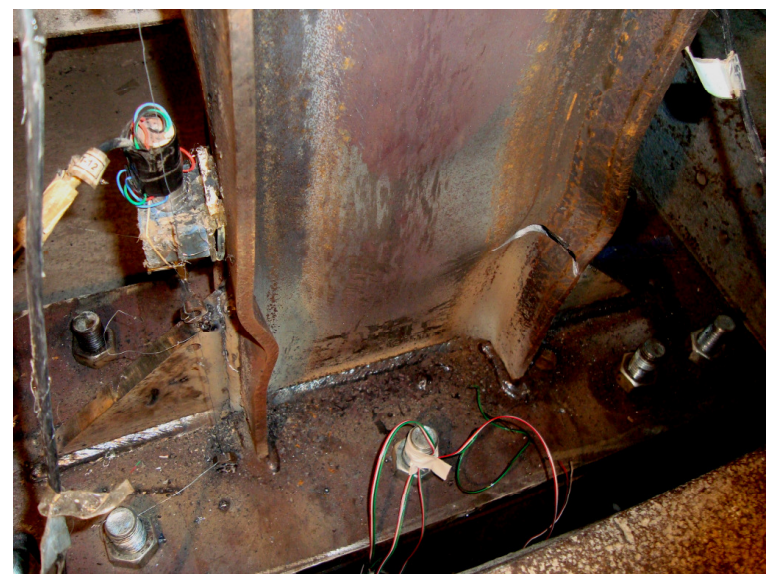

Figure 10. Plastification of specimen TBeam-2 and fracture of the section above the stiffeners after Test- 12

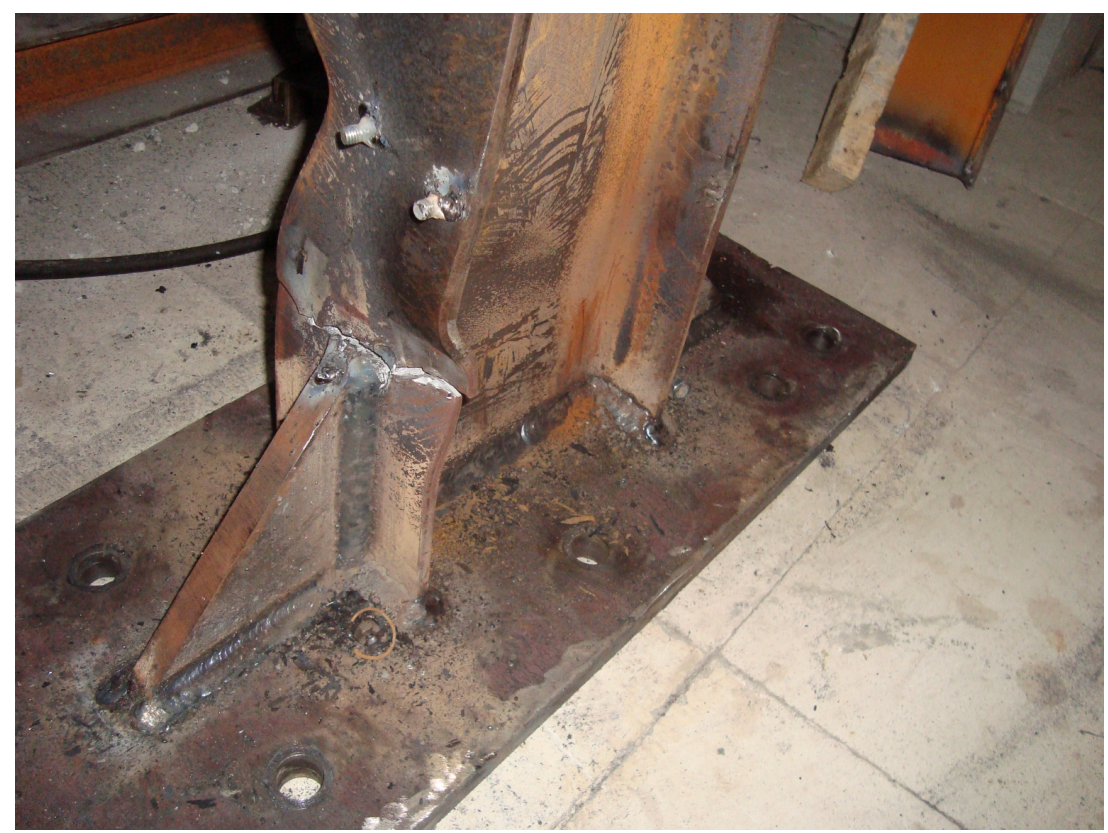

Figure 11. Plastification of specimen TBeam-4 after test -7 . 


\subsection{Measured response in terms of plastic rotations at the plastic hinge.}

Figure 12, depicts the full loading sequence in terms of horizontal displacement at the top of the specimen that could be deduced from the employed instrumentation as directly linked with the elastic/plastic rotation at the zone of the specimen extending at a length of $100 \mathrm{~mm}$ above the stiffeners of the IPE 240 beam (see section 2.3 and figures 2 and 6). The corresponding development of the plastic rotation with time in this plastic hinge region is shown in figure 13. The imposed loading history in terms of plastic hinge rotation and the corresponding horizontal displacement at the top of specimen Tbeam-4 is shown in figure 15 and 14.

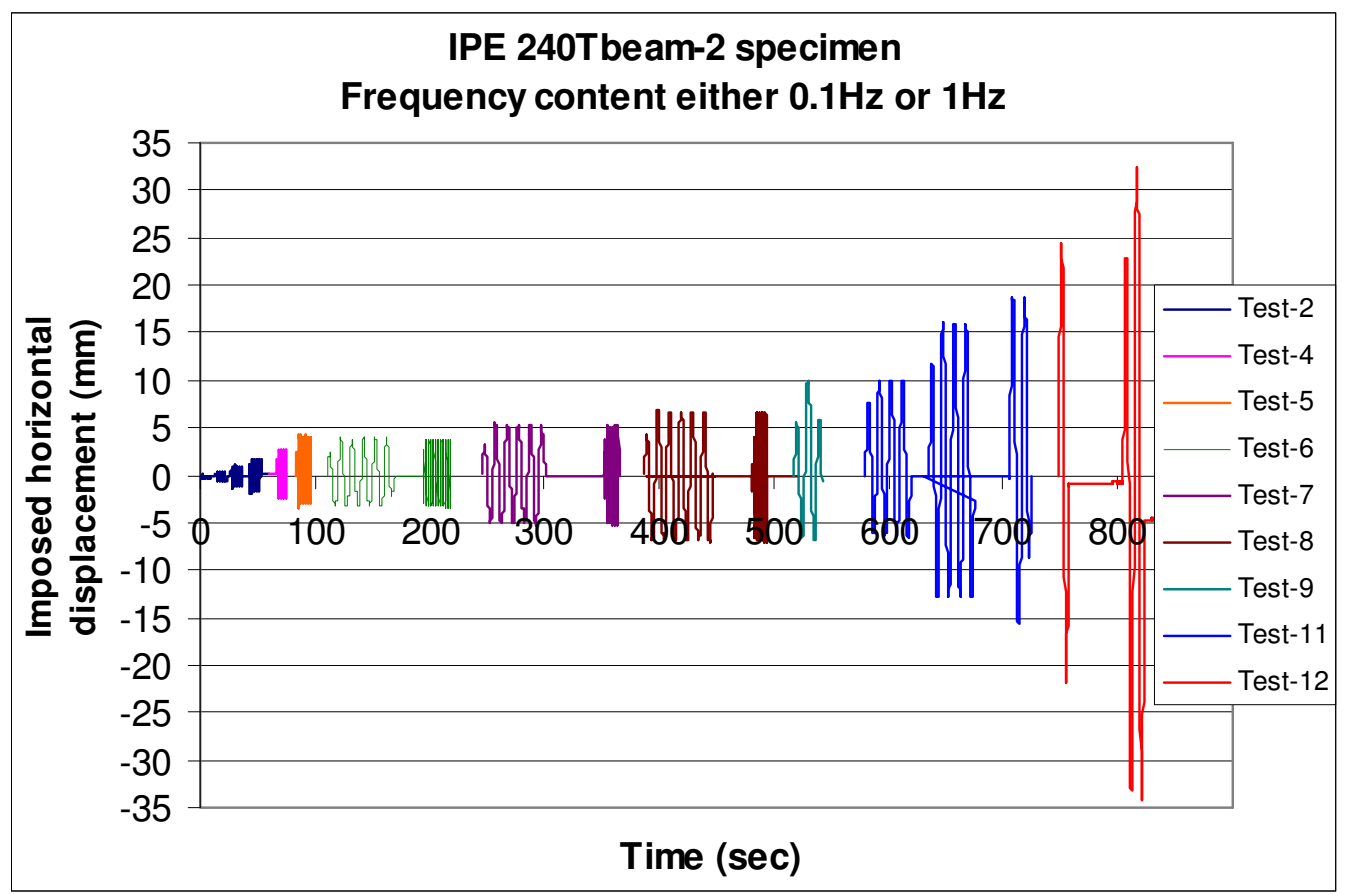

Figure 12: Horizontal displacement at the top of specimen Tbeam-2 due to the plastic rotation at the plastic hinge region.

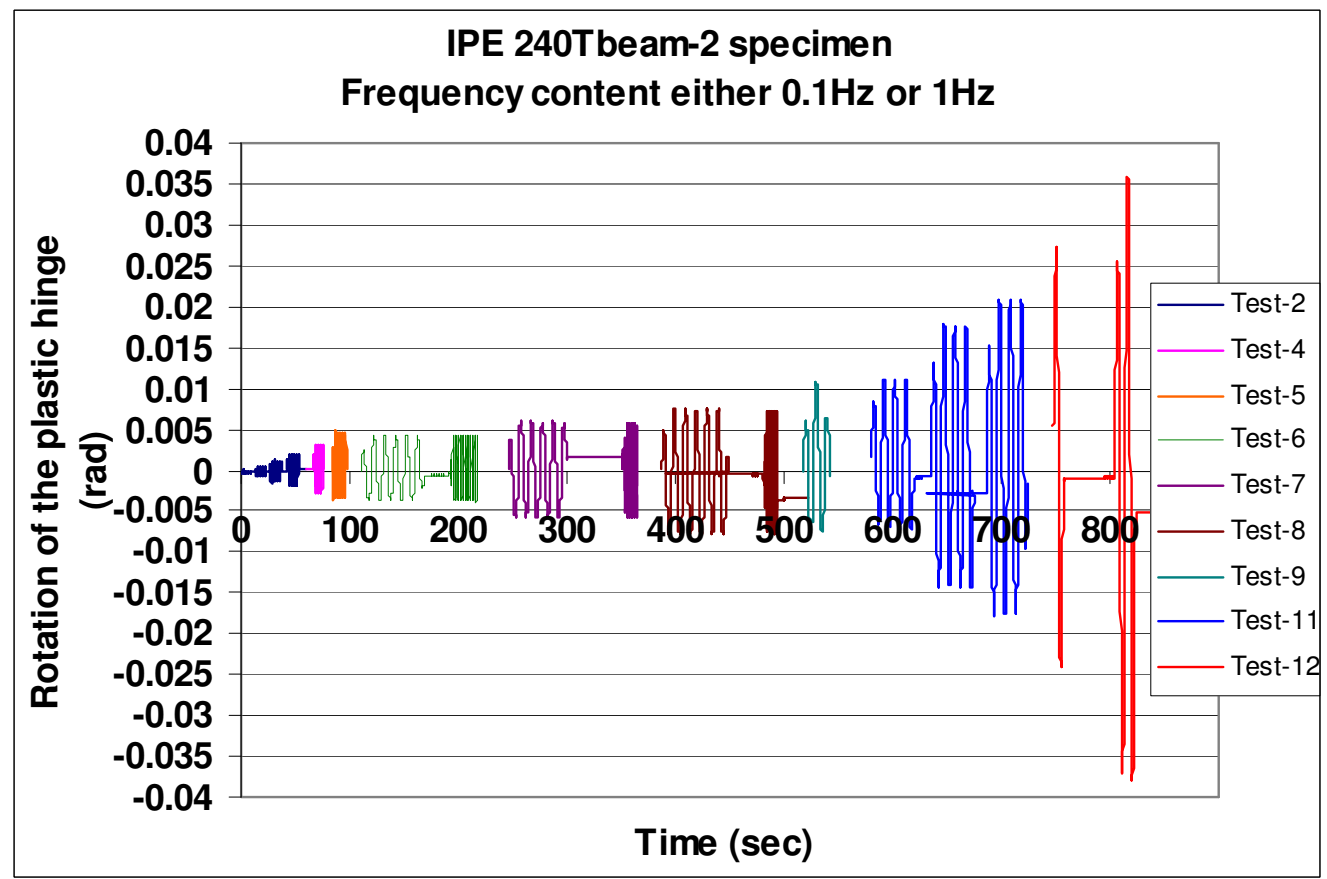

Figure 13: Plastic rotation at the plastic hinge region due to horizontal displacement at the top of specimen Tbeam-2. 


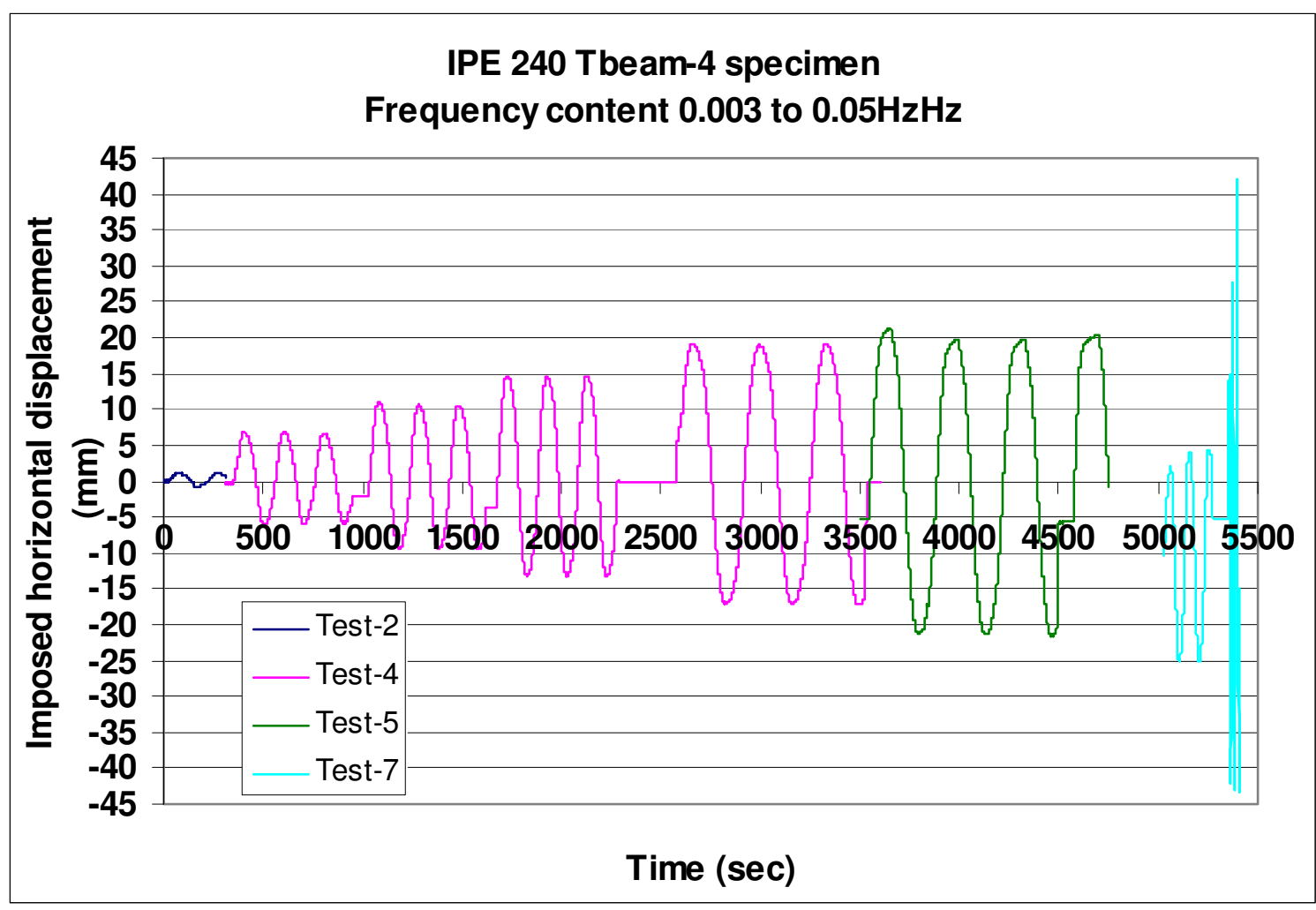

Figure 14: Horizontal displacement at the top of specimen Tbeam-4 due to the plastic rotation at the plastic hinge region.

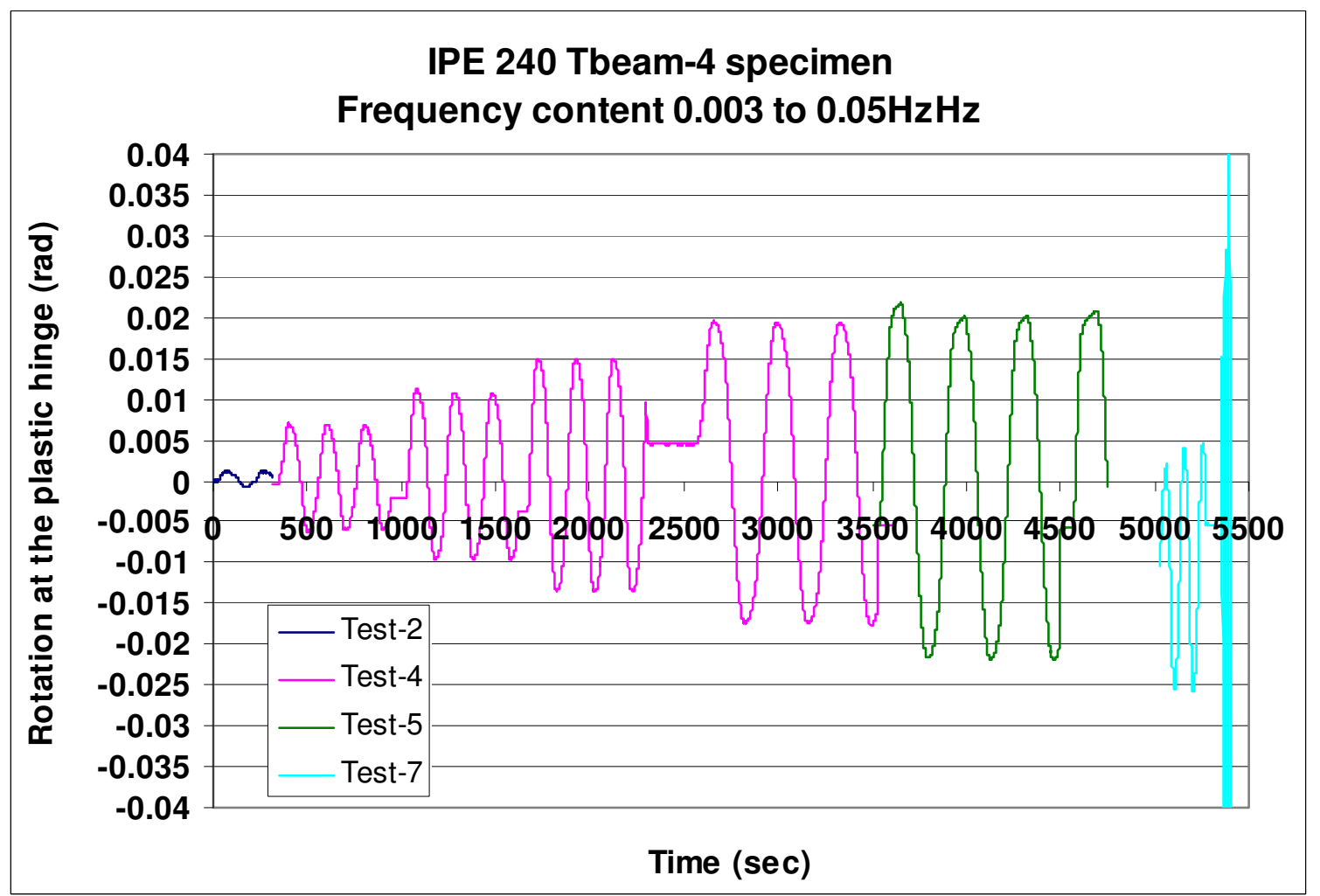

Figure 15: Plastic rotation at the plastic hinge region due to horizontal displacement at the top of specimen Tbeam-4. 
As can be seen in figures 13 and 15 both specimens reach their flexural plastic limit for plastic hinge rotations of the order of 0.023 radians, which represents a value approximately equal to $5 \Theta_{\mathrm{pl}}[13]$, having undergone a considerable number of low-fatigue cycles [10, $13,14]$. Moreover, both specimens failed by developing local instability at the plastic region in the form of flange local buckling and eventually fracture. Figures 16 and 17 portray the observed cyclic response in terms of bending moment and plastic hinge rotation, for specimens Tbeam-2 and Tbeam-4, respectively. The bending moment values were deduced at a section above the stiffeners from the measured horizontal load.

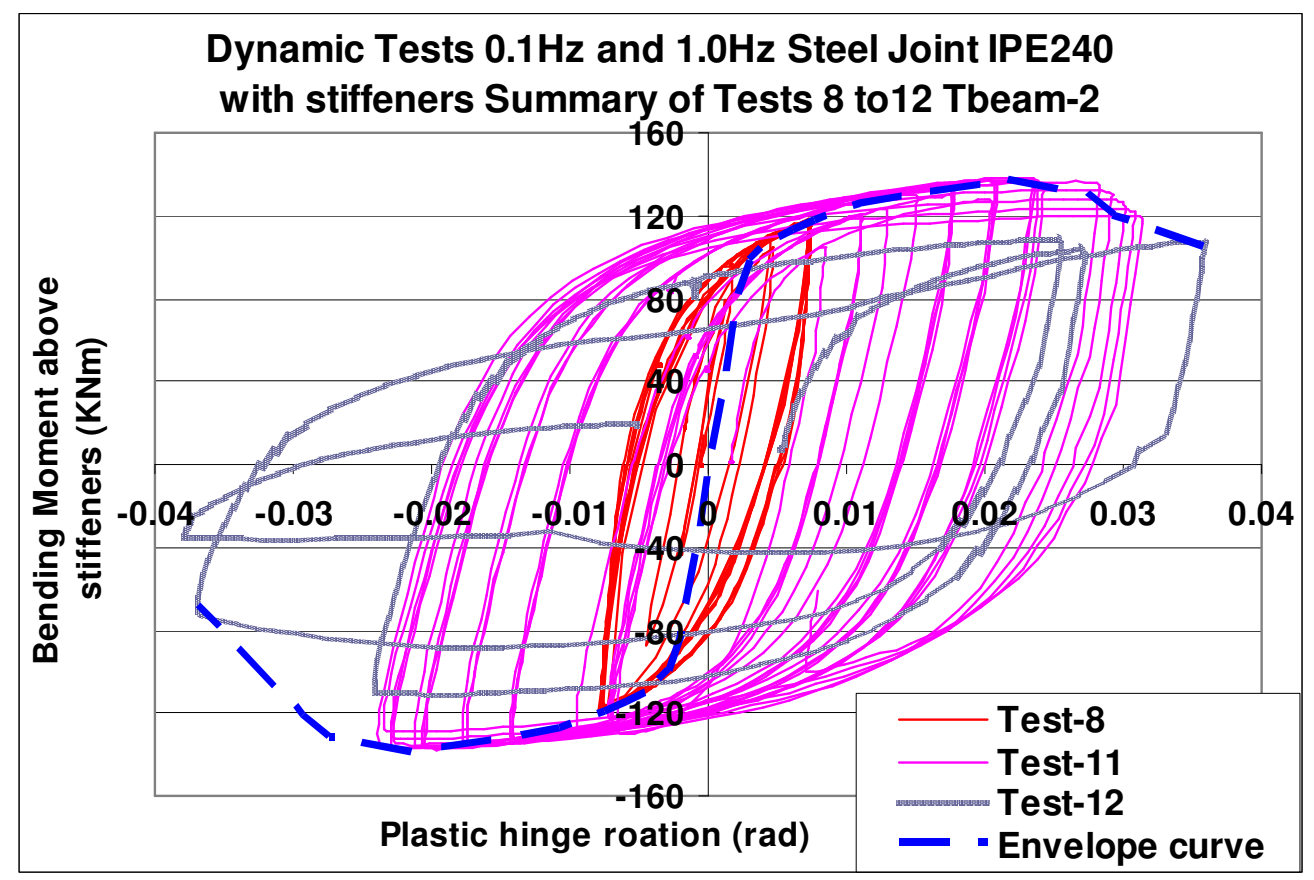

Figure 16: Measured cyclic response of the plastic hinge for specimen Tbeam-2.

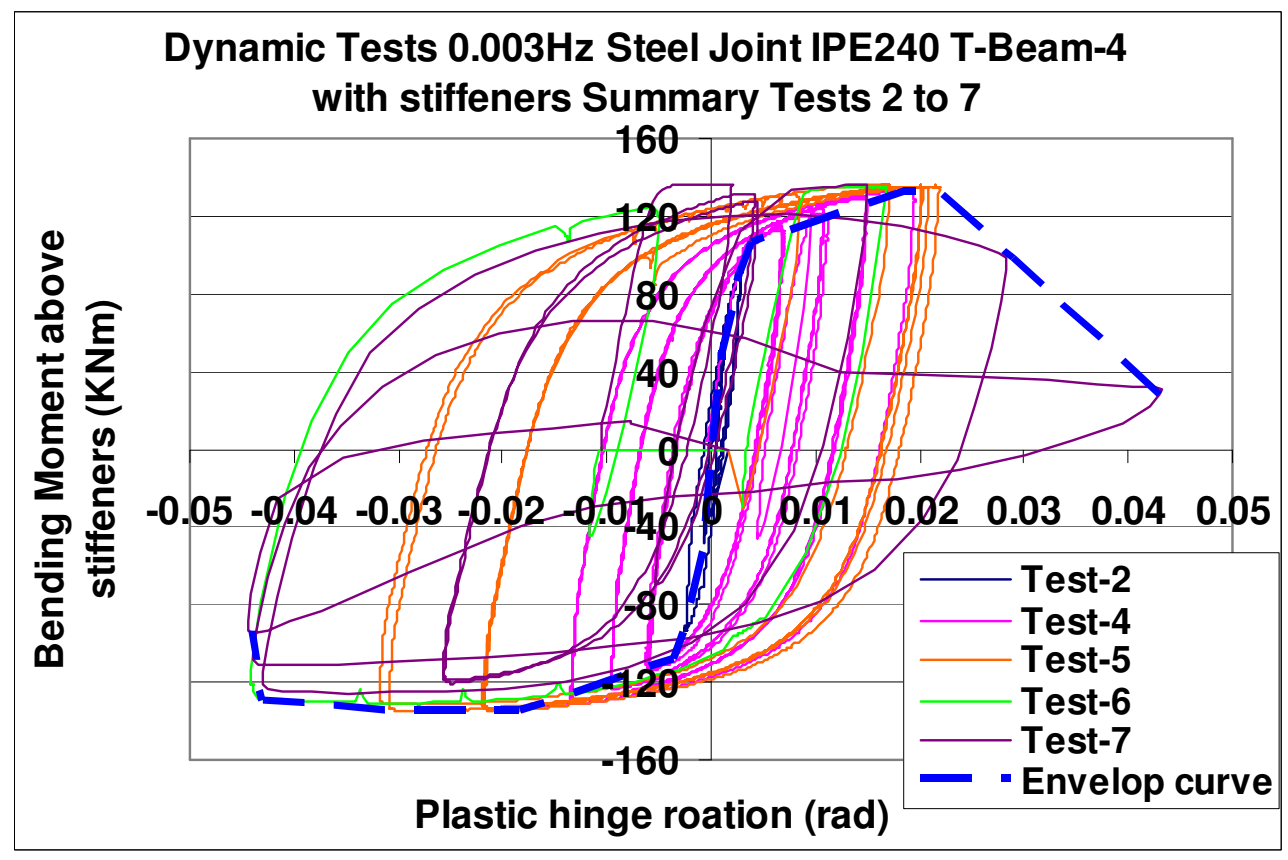

Figure 17: Measured cyclic response of the plastic hinge for specimen Tbeam-4. 
In both figures 16 and 17 a corresponding envelop curve is also plotted with a dashed blue line. As can be seen, both specimens developed large cycles of stable plastic response before reaching their failure mode thus dissipating considerable energy in terms of hysteretic damping. Figure 18 depicts this hysteretic cyclic response through the corresponding envelop curves. As can be seen from the comparison of these two envelop curves in figure 18, both specimens performed in a quite similar way in terms of initial stiffness, maximum flexural bearing capacity and plastic hinge rotation corresponding to that flexural capacity. The measured flexural capacity is of the order of $138 \mathrm{KNm}$ and $134 \mathrm{KNm}$ for specimens Tbeam-2 and Tbeam-4, respectively. Similarly, the plastic hinge rotation values corresponding to these flexural capacity values are in the range approximately 0.020 to 0.025 radians for both specimens. Consequently, it can be concluded that the variation in the frequency content that was employed in the loading sequence for specimen Tbeam-2, which was somehow different from the loading sequence of specimen Tbeam-4 (see figures 13 and 15), did not have any major influence on the observed cyclic response and limit state performance for the two studied specimens.

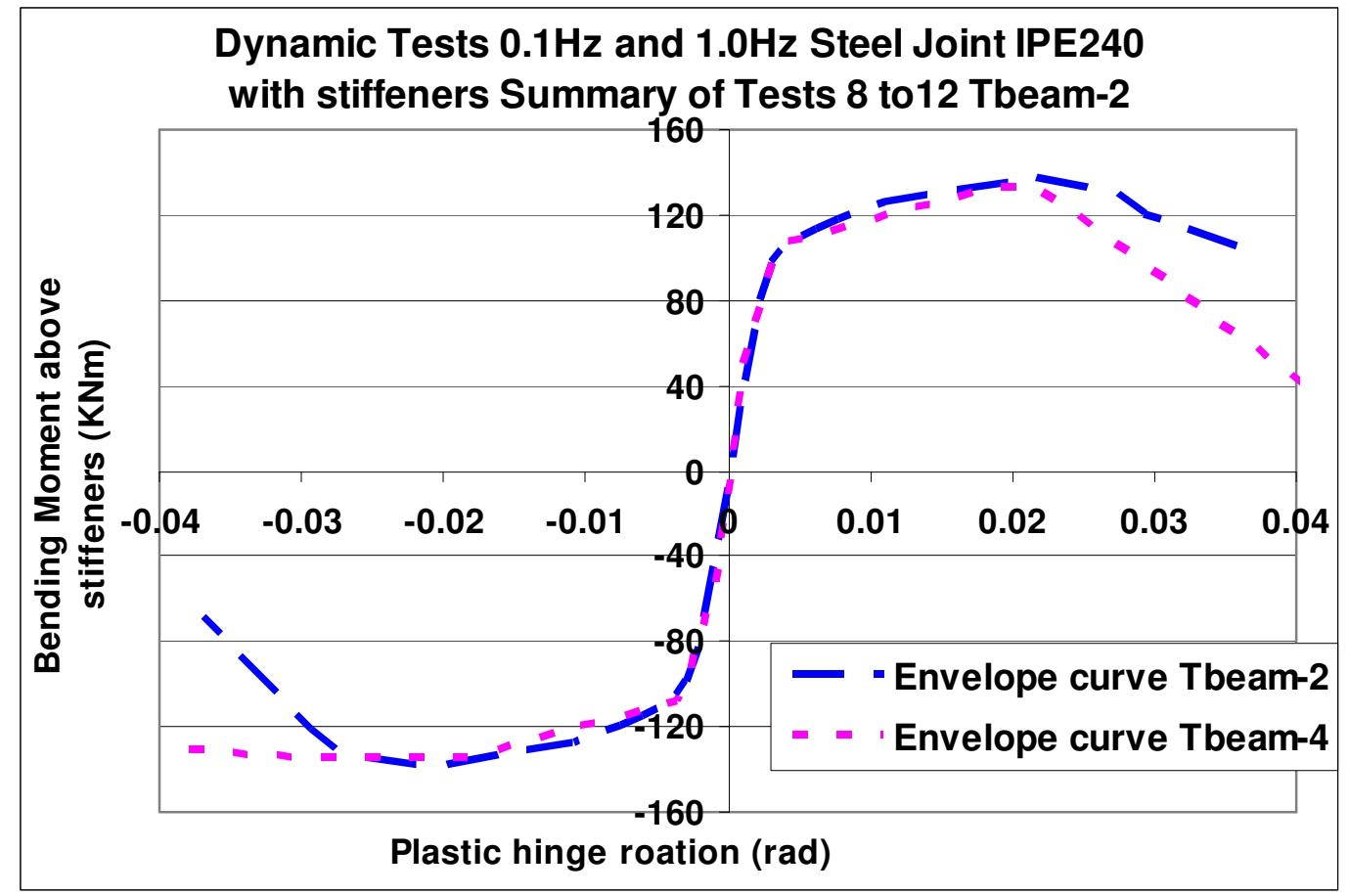

Figure 18: Measured cyclic response of the plastic hinge in terms of envelop curves for both specimens Tbeam-2 and Tbeam-4.

\section{NUMERICAL SIMULATION OF THE OBSERVED CYCLIC RESPONSE AND PERFORMANCE}

\subsection{Numerical modeling.}

The commercial software ABAQUS [16] was utilized in this numerical simulation effort. In doing so, the IPE 240 beam part of the experimental sequence was reproduced through the finite element representation as accurately as possible. This is shown in figure 19 whereby it can be seen that the numerical simulation extended all the way from the top section where the horizontal load was imposed to the bottom horizontal section where the IPE 240 beam was welded to the base plate. Moreover, the triangular stiffeners with their actual thickness and 
geometry were also included in this numerical simulation. The whole surface of the IPE 240 and the stiffeners at the bottom level where the specimens were welded to the base plate was considered to be represented with absolute fixity conditions in the numerical simulation. Moreover, in one set of numerical simulation the specimen was free to displace horizontally in the out-of-the loading plane direction whereas in another set of numerical analyses this possibility of out-of the loading plane horizontal displacement was constrained. The discretization scheme that was adopted was considerably fine in an effort to be able to catch numerically the observed local modes of buckling of the flanges in the plastic hinge region.

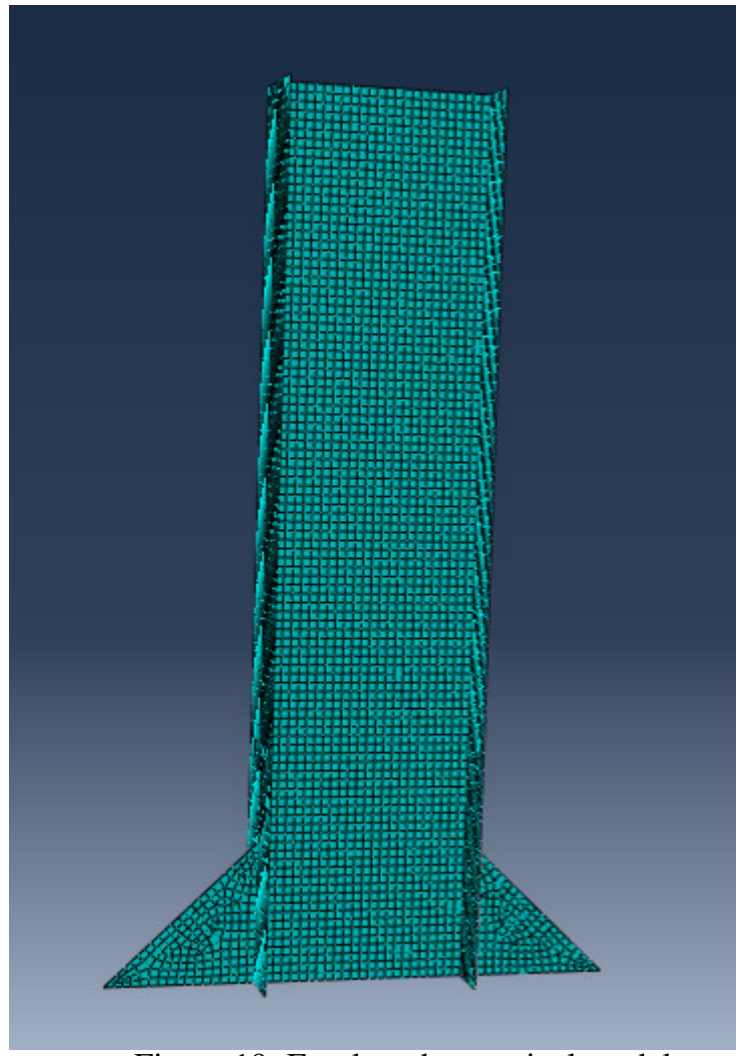

Figure 19: Employed numerical model.

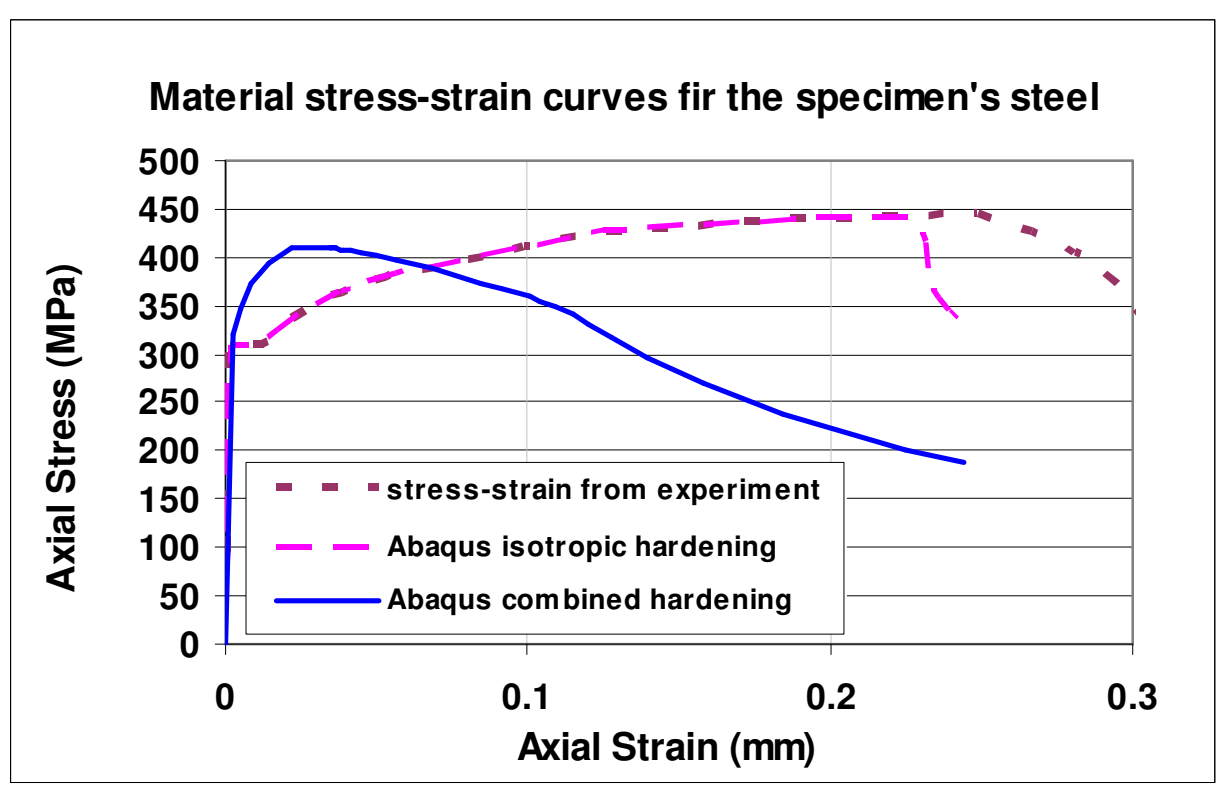

Figure 20: Employed material stress-strain curves 


\subsection{Material modeling}

In the framework of the numerical investigation a parametric study was conducted that focused on the way the steel material properties were introduced in the numerical simulation. Two different approaches were tried. According to the first approach the measured tensile steel properties (see section 2.4) were introduced in the numerical simulation through the Abaqus isotropic hardening parameters whereas in the second approach the measured properties were modified accordingly in order to be introduced in the numerical simulation through the ABAQUS combined hardening material model [16]. This is depicted in figure 20. The measured small differences in the thicknesses and tensile stress-strain behaviour between the first specimen (specimen 1, e.g.Tbeam-2) and the second specimen (specimen 2, e.g. Tbeam-4) were also included in this parametric study. In what follows selected results will be presented.

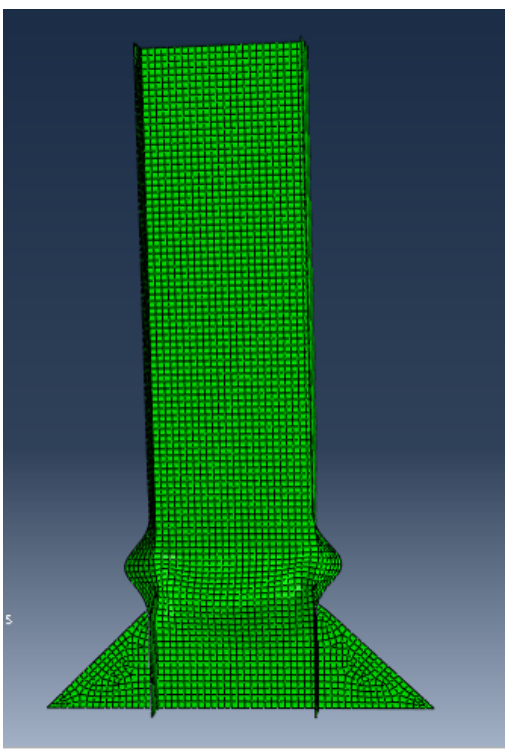

Figure 21: Failure mode of specimen 1 predicted by ABAQUS (see also figures 9 and 10)

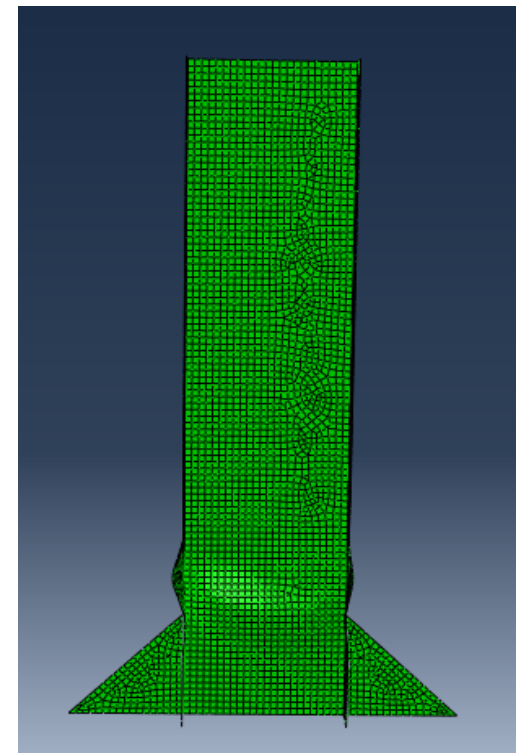

Figure 22 : Failure mode of specimen 2 predicted by ABAQUS (see also figure 11)

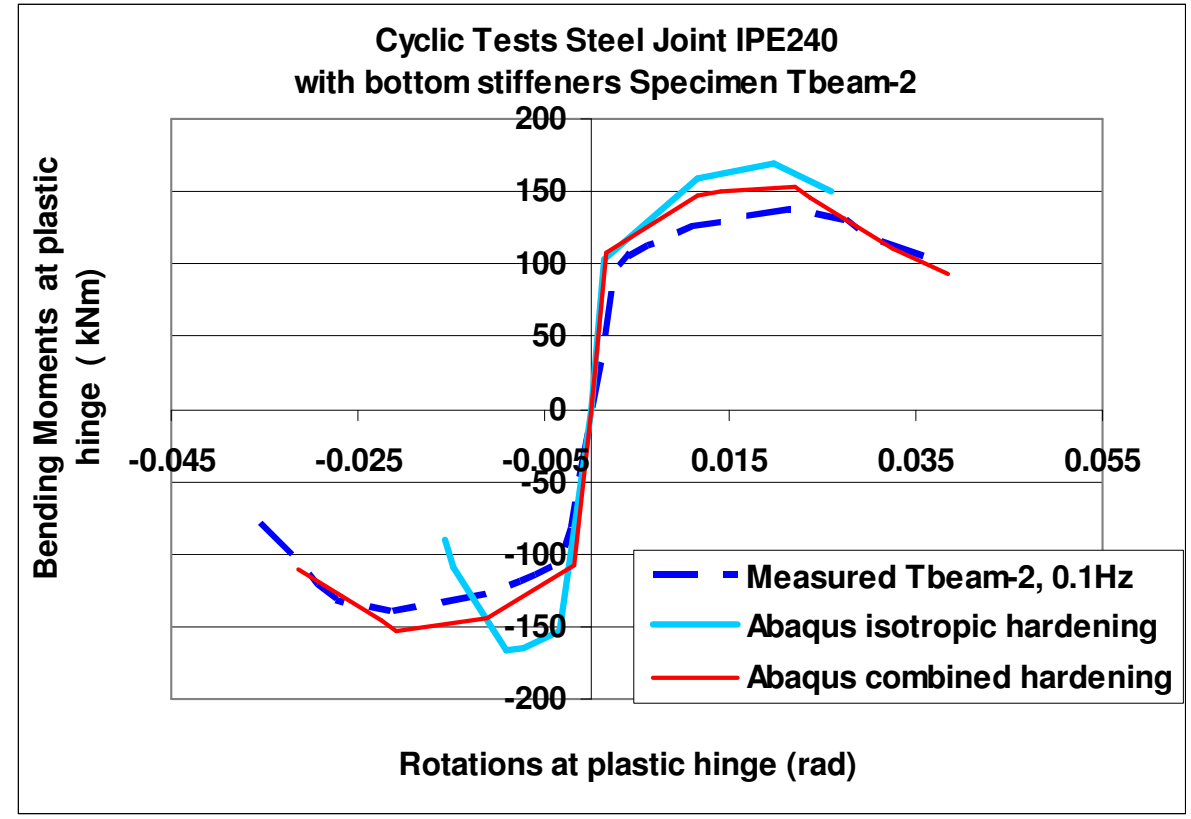

Figure 23: Predicted cyclic response of the plastic hinge in terms of envelop curve for specimen Tbeam-2 together with the measured response. 


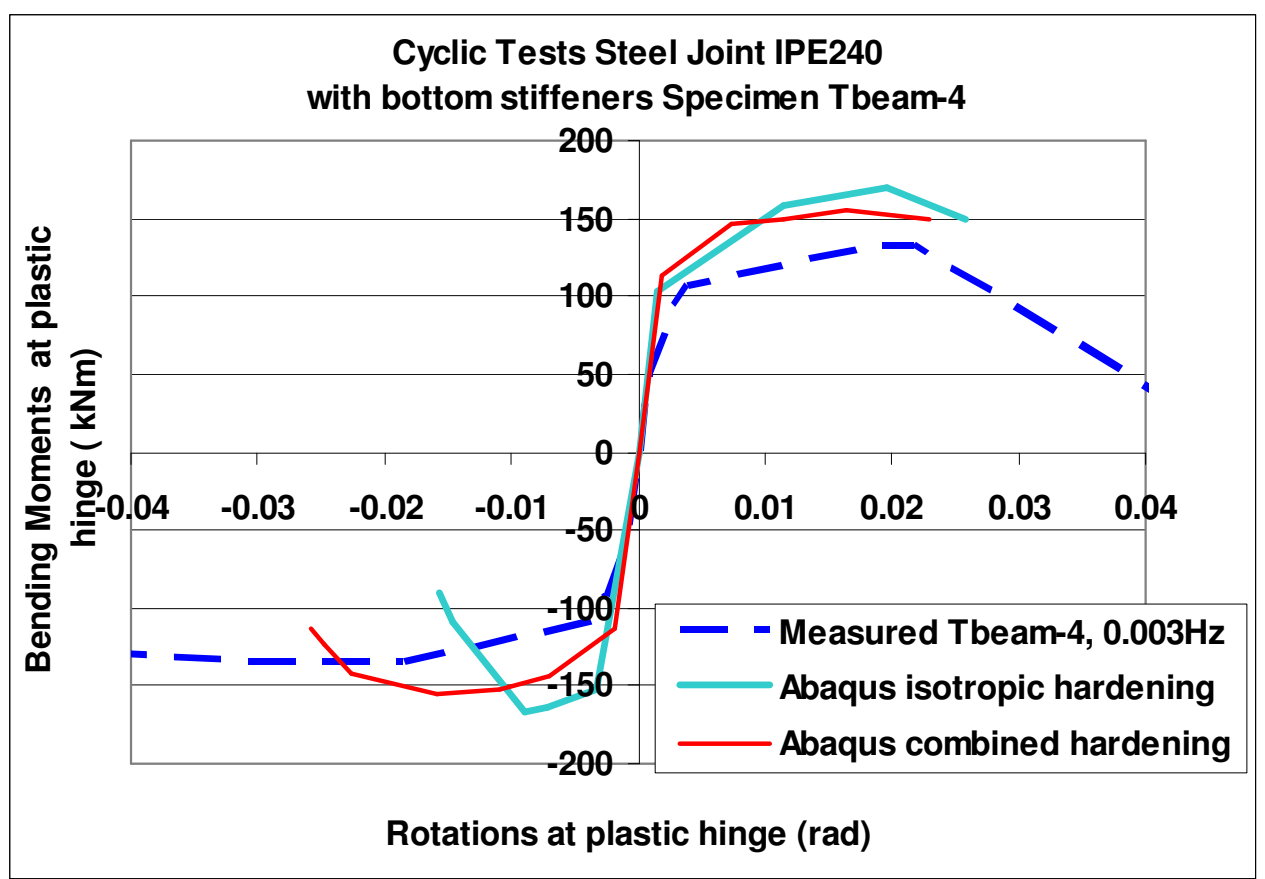

Figure 24: Predicted cyclic response of the plastic hinge in terms of envelop curve for specimen Tbeam-4 together with the measured response.

\subsection{Numerical results from the parametric study}

Figures 21 and 22 depict the predicted failure mode of specimen 1 and specimen 2, respectively, as predicted by the described numerical simulation (ABAQUS [16], see also figures 9 and 10 and 11). These predicted modes of local buckling of the IPE 240 flanges near the beam-to-column joint are in good agreement with the corresponding performance of the tested specimens. Figures 23 and 24 compare the envelop curves predicted by the numerical simulation for specimens Tbeam-2 and Tbeam-4, respectively, with the corresponding envelop curves of the measured cyclic response for the same specimens. The predicted response was obtained by using either the isotropic or the combined hardening material model. As can be seen from figures 23 and 24 the introduction of the combined hardening material model yields a better agreement with the measured response than the isotropic hardening material model.

The next stage in the numerical study was to systematically vary the various parameters that define the combined hardening material model. As could be seen, the variation of these parameters influences important aspects of the cyclic response such as the predicted flexural capacity as well as the plastic rotation range that the cyclic response remains stable in terms of bending moment and the plastic rotation range that the response deteriorates in terms of bending moment and the rate of this deterioration.

This is depicted in figure 25 with the described numerical simulation applied for representing the cyclic response of specimen Tbeam-2. This resulted from an additional parametric study whereby the characteristic parameters that define the combined hardening model were varied within realistic limits in order to study the sensitivity of the obtained cyclic response to the values of these parameters. These parameters are $\boldsymbol{\sigma}_{0}$ (yield stress at zero equivalent plastic strain), $\boldsymbol{C}$ and $\boldsymbol{\gamma}$ (material constants) Nip et al. [17] followed a certain experimental process whereby these parameters were defined in an explicit way through measurements obtained from tests of steel samples. The values that were found by Nip et al. [17] were utilized in the parametric study that was performed in the present work. Selective results from this paramet- 
ric study are presented in figure 25 whereby the value of parameter $\boldsymbol{\sigma}_{0}$ (yield stress at zero equivalent plastic strain) is in one case $250 \mathrm{MPa}$ and in the other case $308 \mathrm{MPa}$ whereas the values of the material constants $\boldsymbol{C}$ and $\boldsymbol{\gamma}$ remain the same for both cases. These two cases were selected from a considerable number of other trial cases. This selection was based on the fact that the resulting response exhibited the best possible comparison with the measured response of specimen Tbeam- 2 as shown in figure 26.

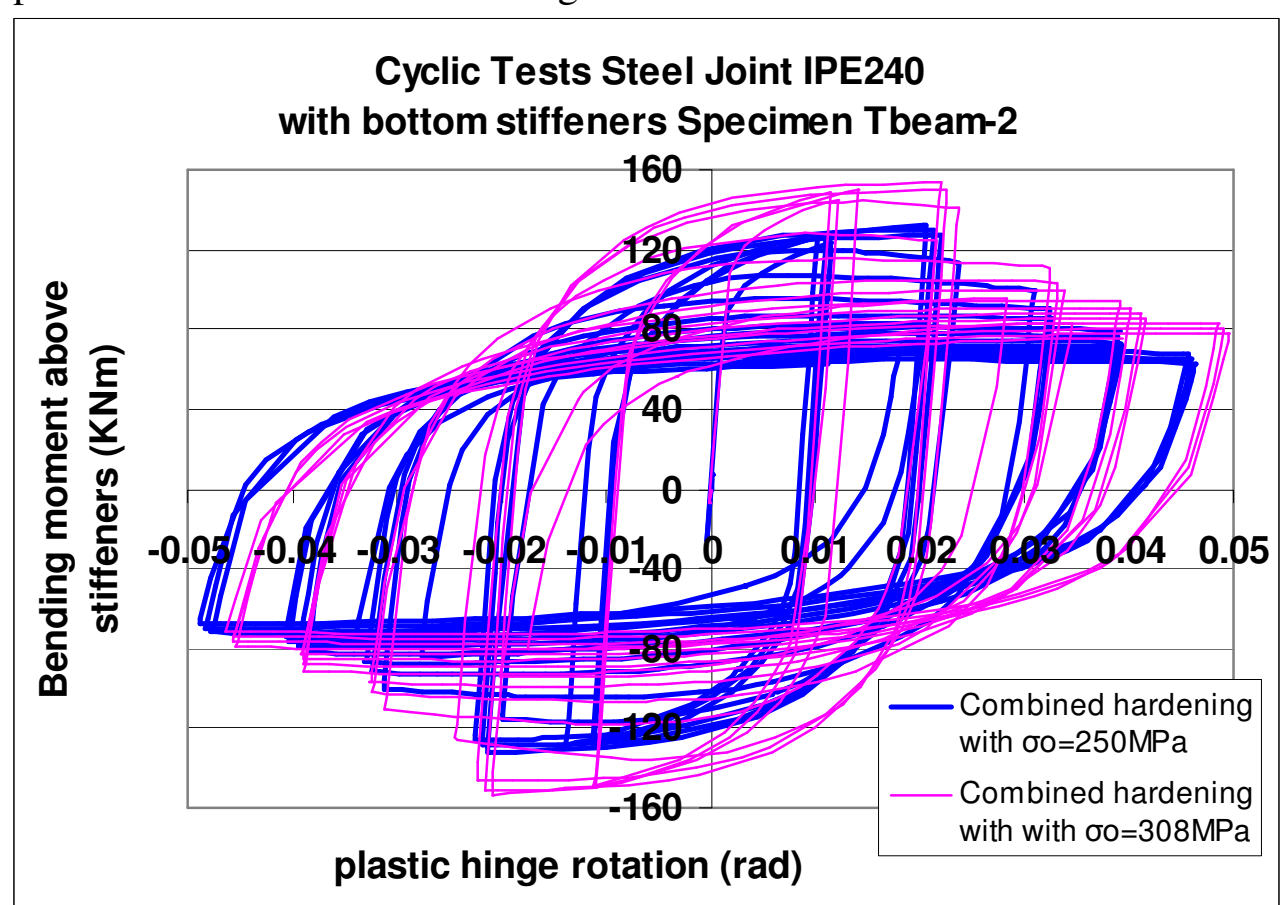

Figure 25: Predicted cyclic response of the plastic hinge in terms of bending moment versus plastic hinge rotation for specimen Tbeam-2 employing two variations of the combined hardening material model.

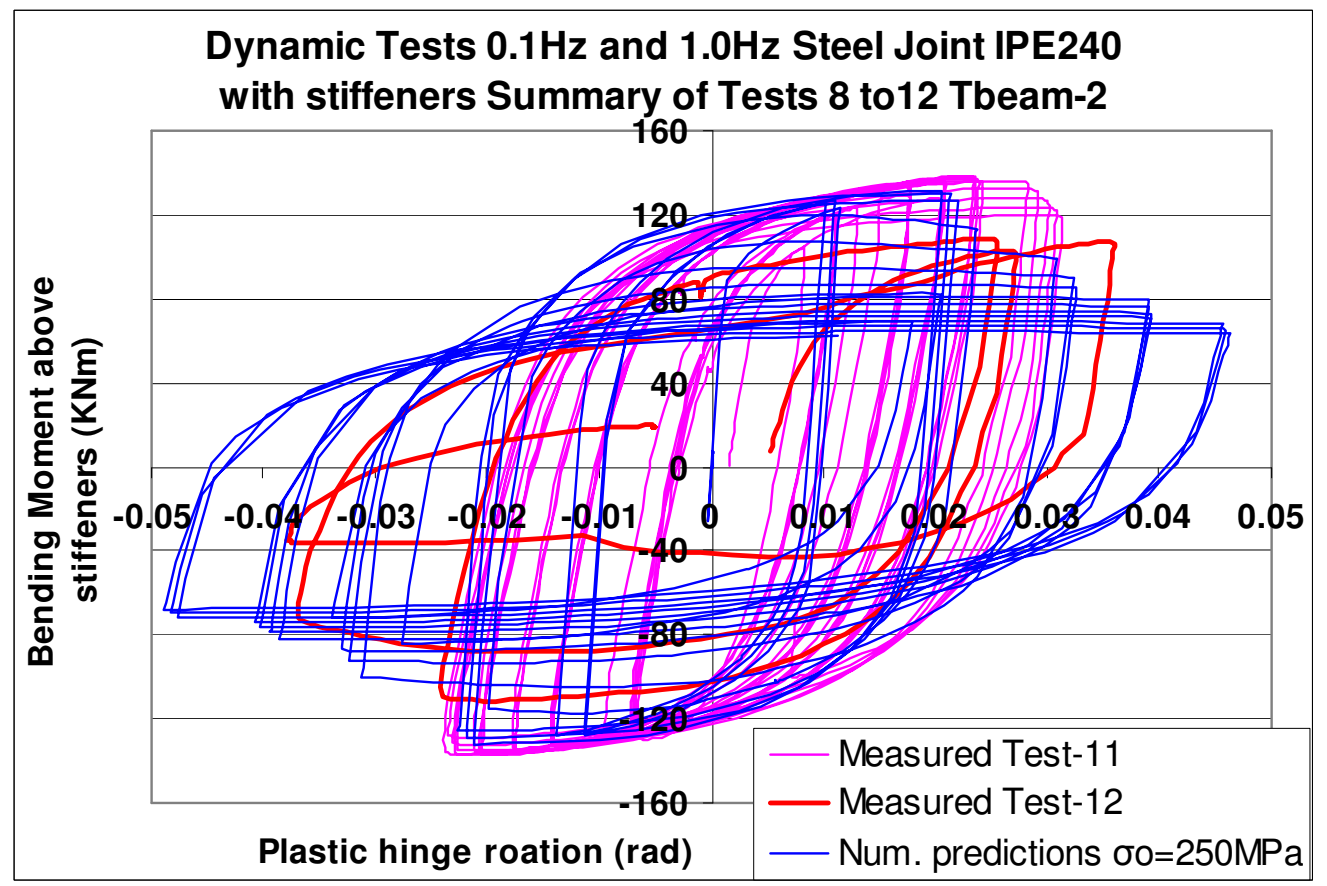

Figure 26: Measured cyclic response of the plastic hinge in terms of bending moment versus plastic hinge rotation for specimen Tbeam-2 and comparison with the best fitted numerical predictions employing the combined hardening material model. . 
As can be seen in figure 25, the variation of the value of the parameter $\boldsymbol{\sigma}_{0}$ (yield stress at zero equivalent plastic strain) from 250 to 308 resulted in considerable variation of the predicted maximum value of the bending moment. In addition, as can be seen in figure 26, a reasonably good agreement could be obtained between the measured in specimen Tbeam- 2 and the predicted cyclic response in terms of maximum flexural capacity, dissipation characteristics and deterioration of the flexural capacity for plastic hinge rotation values larger than 0.023 radians, when the value of the parameter $\boldsymbol{\sigma}_{0}$ (yield stress at zero equivalent plastic strain) is set equal to $250 \mathrm{MPa}$.

\section{CONCLUSIONS}

- The seismic performance of a steel beam-to-column connection was studied by subjecting two specimens of such steel beam-to-column connection specimens to imposed cyclic displacements with a variety of low to medium range strain rates. The tested specimens were constructed with prototype steel sections welded in a typical fashion.

- Both these specimens performed in a quite similar way in terms of initial stiffness, maximum flexural bearing capacity and plastic hinge rotation corresponding to that flexural capacity. Both specimens developed local flange buckling modes of failure for plastic hinge rotation values approximately equal 0.023 radians, which represents a value approximately equal to $5 \Theta_{\mathrm{pl}}$. For values of plastic hinge rotation higher than 0.023 radians the deterioration of the flexural capacity became substantial for both specimens as the local flange buckling mode of failure prevailed.

- The consequence of the previous observation is that the employed strain rate variation in the used loading sequence of specimen Tbeam-2 from that of the loading sequence of specimen Tbeam-4 did not have any major influence on the observed cyclic response and limit state performance between these two studied specimens. However, this is a partial conclusions based on rather limited data. Therefore more research is needed to further investigate the strain rate influence on the beam-to-column connections earthquake performance.

- The numerical simulation of the tested specimens utilizing the capabilities of a commercial software package yielded reasonable good agreement between both the measured and predicted cyclic response in terms of maximum flexural capacity, dissipation characteristics and deterioration of the flexural capacity for plastic hinge rotation values larger than 0.023 radians. Moreover, the numerical simulation also yielded realistic predictions of the limit state local buckling of the flanges of the used IPE 240 cross section thus exhibiting good agreement with the observed performance. However, it is of utmost importance to have realistic estimates of the values of the parameters are $\boldsymbol{\sigma}_{0}$ (yield stress at zero equivalent plastic strain), $\boldsymbol{C}$ and $\boldsymbol{\gamma}$ (material constants) which define the steel combined hardening material behaviour. 


\section{ACKNOWLEDGEMENTS}

The authors would like to acknowledge the assistance of M. Theofanous, Dr. Civil Engineer, Lecturer at the Department of Civil Engineering of the University of Birmingham, for all his valuable advice related to the numerical simulation. The technical assistance of $\mathrm{T}$. Koukouftopoulos in preparing the experimental arrangement is also gratefully acknowledged.

\section{REFERENCES}

[1] Akiyama H. Earthquake-Resistant Limit-State Design for Buildings. University of Tokyo Press: Tokyo, 1985.

[2] Anastasiadis A., Mosoarca M., Gioncu V., Mazzolani F. M., "Some thoughts for the prediction of the local inelastic capacity of MRF subjected to seismic actions", $8^{\text {th }}$ STESSA Conf. on the Behavior of Steel Structures in Seismic Areas, Tongji Univ., Songhai China, July 2015.

[3] Anastasiadis A, Gioncu V, Mazzolani FM. "New trends in the evaluation of available ductility of steel members." Behavior of Steel Structures in Seismic Areas STESSA 2000; 3-26.

[4] Anderson, James C., Nastar Navid, Rojas Fabian, "Low-Cycle Fatigue Effects on Steel Moment Frames", Safety, Reliability and Risk of Structures, Infrastructures and Engineering Systems - Furuta, Frangopol \& Shinozuka (eds)(C 2010 Taylor \& Francis Group, London, ISBN 978-0-415-47557-0

[5] Ballio Giulio \&. Castiglioni, Carlo A, "Seismic Behaviour of Steel Sections", J. Construct. Steel Research 29 (1994) 21-54

[6] Castiglioni Carlo A., Mouzakis Harris P.,. Carydis Panayotis Gr. (2007): "Constant and Variable Amplitude Cyclic Behavior of Welded Steel Beam-to-Column Connections", Journal of Earthquake Engineering, 11:876-902, 2007, 11:6, 876-902, doi.org/10.1080/13632460601188027.

[7] Clark P, Frank K, Krawinkler H, Shaw R. Protocol for fabrication, inspection, testing, and documentation of beam-column connection tests and other experimental specimens. SAC Steel Project Background Document, Report No. SAC/BD-97/02, 1997.

[8] El Hassouni A, Plumier A., Cherrabi A. "Experimental and numerical analysis of the strain-rate effect on fully welded connections", Journal of Constructional Steel Research (2010), doi 10.1016/j.jcsr.2010.09.02

[9] Federal Emergency Management Agency. Interim testing protocols for determining seismic performance characteristics of structural and nonstructural components. FEMA 461 Draft Document, Applied Technology Council, Redwood City, CA, 2007.

[10] Gioncu V, Petcu D. "Available rotation capacity of wide-flange beam and beamcolumns part 1, 2.”, Journal of Constructional Steel Research 1997; 43: 161-244.

[11] Krawinkler H. Loading histories for cyclic tests in support of performance assessment of structural components. The 3rd International Conference on Advances in Experimental Structural Engineering, San Francisco, 2009. 
[12] Lamarche Charles-Philippe and Tremblay Robert, "Seismically induced cyclic buckling of steel columns including residual-stress and strain-rate effects", Journal of Constructional Steel Research 67 (2011) 1401-1410, doi:10.1016/j.jcsr.2010.10.008

[13] Lee Kyungkoo and Stojadinovic Bozidar, "Low-cycle fatigue limit on seismic rotation capacity for US steel moment connections", 13th World Conference on Earthquake Engineering, Vancouver, B.C., Canada, August 1-6, 2004, Paper No. 90.

[14] Vayas I. "Investigation of the cyclic behavior of steel beams by application of low-cycle fatigue criteria." Behavior of Steel Structures in Seismic Areas STESSA 1997; 350-357.

[15] Yu Jiao, Satoshi Yamada, Shoichi Kishiki and Yuko Shimada, "Evaluation of plastic energy dissipation capacity of steel beams suffering ductile fracture under various loading histories" Earthquake Engng Struct. Dyn. 2011; 40:1553-1570.

[16] Hibbitt, Karlsson, Sorensen. Inc. ABAQUS user's manual volumes I-V and ABAQUS CAE manual. Version 6.10.1. Pawtucket, USA; 2010.

[17] Nip, K.H., Gardner L., Davies C.M., Elghazouli A.Y., "Extremely low cycle fatigue tests on structural carbon steel and stainless steel", Journal of Constructional Steel Research 66 (2010) 96-110, doi:10.1016/j.jcsr.2009.08.004 